\title{
Caratteristiche clinico-patologiche, gestione clinica e prognosi dell'insulinoma maligno: studio multicentrico italiano
}

\author{
Alessio Veltroni ${ }^{1} \cdot$ Elisa Cosaro ${ }^{1} \cdot$ Maria Vittoria Davì ${ }^{1}$
}

Accettato: 1 agosto 2020 / Pubblicato online: 17 marzo 2021

(c) The Author(s) 2021

Sommario L'insulinoma maligno è un tumore neuroendocrino pancreatico estremamente raro ed è associato a una severa sindrome ipoglicemica che impatta negativamente sulla qualità di vita e sulla sopravvivenza dei pazienti affetti. La gestione terapeutica dell'insulinoma maligno è complessa sia per il controllo delle crisi ipoglicemiche, sia per il controllo della crescita tumorale. La sindrome ipoglicemica rappresenta una sfida terapeutica per l'endocrinologo in quanto spesso non è responsiva alla terapia medica sintomatica, in particolare al diazossido utilizzato in monoterapia o associato agli analoghi della somatostatina. Everolimus ha un ruolo nel trattamento delle crisi ipoglicemiche refrattarie da insulinoma maligno sia per l'azione di inibizione del rilascio di insulina che di insulino-resistenza. La chirurgia con approccio curativo dell' insulinoma maligno è raramente perseguibile a causa della diffusione metastatica, mentre la chirurgia a scopo di debulking può essere presa in considerazione in casi selezionati sia per il controllo sintomatico sia perché può aumentare l'efficacia delle terapie sistemiche o locoregionali. La terapia radiometabolica con analoghi caldi della somatostatina rappresenta un'opzione terapeutica nei pazienti con tumori a elevata espressione dei recettori della somatostatina sia per il controllo della sintomatologia che della crescita tumorale, sebbene l'esperienza negli insulinomi maligni sia piuttosto scarsa. Data la rarità della malattia,

Proposto da Marco Gallo.

Informazioni Supplementari La versione online contiene materiale supplementare disponibile su

https://doi.org/10.1007/s40619-021-00843-2.

M.V. Davì

mariavittoria.davi@aovr.veneto.it

1 U.O.S. di Endocrinologia, Policlinico G.B. Rossi, ENETS Center of Excellence, Azienda Ospedaliera Universitaria Integrata, Verona, Italia sono disponibili in letteratura solo descrizioni di singoli casi o studi condotti su casistiche limitate; pertanto, è difficile stabilire la sequenza terapeutica più efficace in questi casi. Recentemente è stato condotto uno studio multicentrico italiano, in 13 centri di riferimento, focalizzato sulle caratteristiche clinico-patologiche, sulle modalità di trattamento e sui fattori prognostici che condizionano decorso ed esito dell'insulinoma maligno allo scopo di individuare una strategia terapeutica mirata basata su criteri razionali ed evidenze cliniche. In questa rassegna verranno descritti i principali risultati dello studio che comprende una casistica tra le più ampie finora pubblicate.

Parole chiave Insulinoma maligno - Ipoglicemia · Insulina • Trattamento insulinoma $\cdot$ Prognosi insulinoma

\section{Introduzione}

L'insulinoma è il tumore endocrino funzionante del pancreas (panNET) più frequente; si presenta nella maggior parte dei casi benigno, con un'incidenza di 4 casi per milione l'anno.

Solo nel 10\% dei casi ha un comportamento maligno con metastasi locoregionali e/o a distanza, con insorgenza intorno alla V-VI decade, senza predominanza di sesso [1]. Il quadro clinico è caratterizzato da crisi ipoglicemiche in genere più frequenti e severe rispetto alle forme benigne associate a elevati livelli di insulina e C-peptide. Spesso non è necessario eseguire il test a digiuno per evocare l'ipoglicemia, data la gravità della sintomatologia clinica. La sindrome ipoglicemica è generalmente presente alla diagnosi del tumore, ma può occasionalmente manifestarsi in un panNET metastatico originariamente non funzionante che inizia a secernere successivamente insulina durante il decorrere della 
malattia. Il viraggio verso la forma funzionante è spesso associato a un comportamento aggressivo della neoplasia e a un impatto negativo sulla prognosi [2].

La gestione terapeutica dell'insulinoma maligno è complessa sia per il controllo della sindrome ipoglicemica che per il controllo della crescita tumorale. La sindrome ipoglicemica rappresenta una sfida terapeutica per l'endocrinologo in quanto spesso è refrattaria alla terapia sintomatica, in particolare al diazossido che è il farmaco più utilizzato in prima linea. Gli analoghi della somatostatina (SA) possono essere utilizzati in alternativa o associazione al diazossido con un'efficacia dimostrata in circa il 50\% dei pazienti. Everolimus (EVE) è indicato nella sindrome ipoglicemica refrattaria da insulinoma maligno sia per l'azione di inibizione del rilascio di insulina che di insulino-resistenza [3]. La chirurgia con approccio curativo è raramente perseguibile a causa della diffusione metastatica in particolare al fegato, mentre la chirurgia a scopo di debulking può essere presa in considerazione in casi selezionati sia per il controllo sintomatico sia perché può aumentare l'efficacia delle terapie sistemiche o locoregionali. Tuttavia, l'impatto della chirurgia nel trattamento dei panNET in casistiche comprendenti solo insulinomi maligni è stata finora scarsamente valutata. Un recente studio retrospettivo basato sul registro tumori americano Surveillance, Epidemiology and End Results (SEER) ha incluso 121 pazienti con diagnosi tra il 1973 e 2015 di insulinoma maligno, di cui il $40 \%$ con malattia localizzata, $16 \%$ con metastasi linfonodali e $39 \%$ con metastasi a distanza [4]. Il trattamento chirurgico è stato eseguito nel $64 \%$ dei casi e ha rappresentato, insieme al sesso maschile e assenza di metastasi, un fattore predittivo favorevole di sopravvivenza. Nonostante l'ampia casistica, il limite di questo studio è la mancanza di informazioni riguardanti la presentazione clinica, la diagnosi biochimica e le altre modalità di trattamento al di fuori della chirurgia.

La terapia radiometabolica con analoghi caldi della somatostatina (PRRT) rappresenta un'opzione terapeutica nei pazienti con elevata espressione dei recettori della somatostatina sia per il controllo della sintomatologia che della crescita tumorale, sebbene l'esperienza negli insulinomi maligni sia piuttosto scarsa $[5,6]$.

Data la rarità della malattia, sono disponibili in letteratura solo singole segnalazioni di casi o studi condotti su casistiche limitate, per cui è difficile stabilire la sequenza di trattamento più efficace in questi casi.

Un recente studio retrospettivo multicentrico italiano [7], condotto in 13 centri di riferimento, ha analizzato 31 pazienti affetti da insulinoma maligno seguiti nel periodo 1988-2017 (Tabella 1). Lo studio ha analizzato le caratteristiche clinico-patologiche, le modalità di trattamento e $\mathrm{i}$ principali fattori prognostici che influiscono l'outcome, alla ricerca di una strategia terapeutica mirata, basata su evidenze cliniche. Con l'ausilio di un questionario, i partecipanti hanno descritto le caratteristiche cliniche e istologiche della malattia, l'approccio diagnostico (laboratoristico e strumentale), le terapie utilizzate (medica, oncologica, chirurgica o medico-nucleare) e il follow-up dal punto di vista della sopravvivenza.

\section{Caratteristiche clinico-patologiche}

Lo studio ha incluso 31 pazienti $(61,3 \%$ maschi) affetti da insulinoma maligno con un età media alla diagnosi di $48 \pm$ 15 anni.

In 5 casi $(16,1 \%)$ la sindrome è comparsa dopo anni dalla diagnosi di un NET originariamente non funzionante. Nei restanti 26 casi $(83,9 \%)$ l'ipoglicemia ha condotto alla diagnosi del NET, di cui $11(42,3 \%)$ con un ritardo diagnostico di $32,7 \pm 39,8$ mesi. Nella maggior parte dei casi $(77,5 \%)$ la diagnosi di sindrome ipoglicemica iperinsulinemica è stata posta in corso di ipoglicemia spontanea, mentre solo nel $22,5 \%$ dei casi è stato necessario eseguire un test al digiuno, interrotto per lo più in prima giornata, a dimostrazione della gravità della sindrome ipoglicemica. Per quanto riguarda il grading che si basa sull'indice proliferativo delle neoplasie (Ki-67 o conta mitotica) la maggior parte dei tumori era ben o moderatamente differenziata $[70,8 \%$ G2 (Ki-67 3-20\%), $12,5 \%$ G1 (Ki-67 0-2\%), mentre solo il $16,7 \%$ era G3 (Ki$67 \geq 20 \%$ ). Tutti i tumori G3 erano ben differenziati da un punto di vista morfologico e classificabili come NET secondo la classificazione WHO 2017. L'immunoistochimica per la ricerca di insulina su materiale bioptico è risultata positiva in 11/14 $(78,4 \%)$. La negatività dell'insulina all'immunoistochimica di alcuni insulinomi maligni riflette l'elevato e rapido tasso di secrezione dell'ormone da parte delle cellule tumorali ed è quindi espressione di maggiore aggressività tumorale [8]. Le metastasi erano diffuse in $12 / 31$ pazienti $(31,7 \%)$, epatiche in $13 / 31(41,9 \%)$, solo linfonodali in $6 / 31$ $(19,4 \%)$.

Per quanto riguarda le procedure diagnostiche, la TC è stata la procedura radiologica più utilizzata, seguita dalla RM addominale, con una positività nel $100 \%$ dei casi. Otto casi sono stati sottoposti a Octreoscan, anch'esso positivo nel $100 \%$; la ${ }^{68} \mathrm{Ga}$-DOTA-peptidiPET/TC era positiva in $13 / 14(92,3 \%)$ e la ${ }^{18}$ FDG PET/TC in $8 / 9(88,9 \%)$ dei pazienti che le hanno effettuate. Degli 8 pazienti sottoposti sia a ${ }^{68} \mathrm{Ga}$-DOTA-peptidi-PET/TC che a ${ }^{18}$ F-FDG-PET/TC, in 7 casi entrambe le procedure sono risultate positive, mentre in uno ${ }^{6}{ }^{68} \mathrm{Ga}$-DOTA-peptidi-PET/TC era positiva e la ${ }^{18}$ FDG-PET/TC negativa.

È importante sottolineare l'elevata sensibilità (>90\%) della ${ }^{68} \mathrm{Ga}$-DOTA-peptidi-PET/TC nell'insulinoma maligno rispetto all'insulinoma benigno che, al contrario, presenta recettori della somatostatina in meno del $50 \%$ dei casi. Le caratteristiche clinico-tumorali, i dati di laboratorio e di diagnostica per immagini sono elencati nella Tabella 1. 
Tabella 1 Caratteristiche clinico-patologiche, dati di laboratorio e di diagnostica per immagini dei pazienti affetti da insulinoma maligno [7]

\begin{tabular}{|c|c|}
\hline \multicolumn{2}{|c|}{ Caratteristiche cliniche e anatomo-patologiche ( $N=31$ pazienti) } \\
\hline Sesso & M: $19(61,3 \%), \mathrm{F}: 12(38,7 \%)$ \\
\hline Periodo considerato & $1988-2017$ \\
\hline Età alla diagnosi (media \pm DS) & $48 \pm 15$ anni \\
\hline $\begin{array}{l}\text { Diagnosi NET e successiva comparsa sindrome } \\
\text { ipoglicemica }\end{array}$ & $\begin{array}{l}5(16,1 \%) \\
\text { intervallo di insorgenza } 46 \pm 35 \text { mesi }\end{array}$ \\
\hline $\begin{array}{l}\text { Diagnosi ipoglicemia e successivo riscontro di } \\
\text { NET }\end{array}$ & $\begin{array}{l}26(83,9 \%) \\
11(42,3 \%) \text { con ritardo diagnostico di } 32,7 \pm 39,8 \text { mesi }\end{array}$ \\
\hline MEN 1 & $1(3,2 \%)$ \\
\hline Dimensioni NET (media \pm DS) & $\begin{array}{l}41 \pm 31 \mathrm{~mm}(>20 \mathrm{~mm}, 65,2 \%) \\
\text { Corpo-coda: } 78,6 \%\end{array}$ \\
\hline Localizzazione pancreatica del tumore & $\begin{array}{l}\text { Testa: } 16 \% \\
\text { Processo uncinato: } 5,4 \%\end{array}$ \\
\hline Grading (Ki-67) & $\begin{array}{l}\text { NET G1 (Ki-67: 0-2\%): 3/24 (12,5\%) } \\
\text { NET G2 (Ki-67 3-20\%): 17/24 (70,8\%) } \\
\text { NET G3 (Ki-67 20\%): 4/24 (16,7\%) }\end{array}$ \\
\hline Presenza di metastasi & $\begin{array}{l}\text { Diffuse } 12 / 31(31,7 \%) \\
\text { Epatiche } 13 / 31(41,9 \%) \\
\text { Linfonodali } 6 / 31(19,4 \%)\end{array}$ \\
\hline Valori di laboratorio & \\
\hline Glicemia minima (media \pm DS) & $34 \pm 8,3 \mathrm{mg} / \mathrm{dl}$ \\
\hline Insulina (media \pm DS) & $67,2 \pm 48,5 \mu \mathrm{U} / \mathrm{ml}$ \\
\hline C-peptide (media \pm DS) & $4,36 \pm 4 \mathrm{ng} / \mathrm{ml}$ \\
\hline CgA elevata & Elevata nel $78,6 \%$ dei casi \\
\hline Esami strumentali & Positività \\
\hline TC e/o RMN & $29 / 29(100 \%)$ \\
\hline Octreoscan & $8 / 8(100 \%)$ \\
\hline${ }^{68} \mathrm{Ga}-\mathrm{PET}-\mathrm{TC}$ & $13 / 14(92,9 \%)$ \\
\hline${ }^{18}$ F-FDG-PET-TC & $8 / 9(88,9 \%)$ \\
\hline
\end{tabular}

\section{Trattamento}

Per quanto riguarda il trattamento, la chirurgia ha rappresentato l'approccio terapeutico più utilizzato ed è stata eseguita in $21 / 31(67,7 \%)$ pazienti $(52,4 \%$ con intento curativo e $47,6 \%$ di debulking). La chirurgia da sola o associata ad altri trattamenti ha determinato un controllo dell'ipoglicemia nel $42,9 \%$ dei pazienti. La maggioranza dei pazienti $(80,6 \%)$ ha eseguito ulteriori linee di trattamento con differenti sequenze, tra cui PRRT (12 casi), EVE (19 casi), chemioterapia ( 8 casi), TACE/TAE/ablazione con radio-frequenza (3 casi), radioterapia ( 2 casi). Un paziente è stato sottoposto a trapianto epatico. La PRRT è stata effettuata in 14 pazienti: in $2 / 14(14 \%)$ come prima opzione insieme a SA, in 8/14 (57\%) come seconda linea di terapia e in 4/14 (28\%) come terza o quarta linea di terapia. Due pazienti hanno ricevuto la PRRT in associazione con EVE a basse dosi per il controllo della sindrome ipoglicemica. Tra i pazienti trattati con PRRT, 6/14 (42,9\%) hanno ottenuto un completo controllo dell'ipoglicemia e 7/14 (50\%) una riduzione degli episodi di ipoglicemia.

\section{Prognosi}

Il follow-up mediano (dalla diagnosi all'ultima valutazione) è stato di 60 mesi. Durante il periodo di follow-up (durata compresa fra 3 e 194 mesi), 11 (35\%) pazienti sono deceduti. Il tasso di sopravvivenza a 5 anni è stato del $62 \%$, mentre quello a 10 anni del $49 \%$. La sopravvivenza globale mediana è stata di 40 mesi (Fig. 1). Non sono state riscontrate differenze statisticamente significative nella prognosi in base a sesso, età alla diagnosi, sito del tumore primitivo e sue dimensioni. Per quanto riguarda il grading tumorale, considerando come cut-off per il Ki-67 il valore del $10 \%$, i pazienti con Ki-67 $\leq 10 \%$ alla diagnosi hanno presentato un tasso di sopravvivenza significativamente maggiore rispetto ai pazienti con Ki-67 > 10\% (a 5 anni 87 vs 43\%, $p=0,03$ ). 


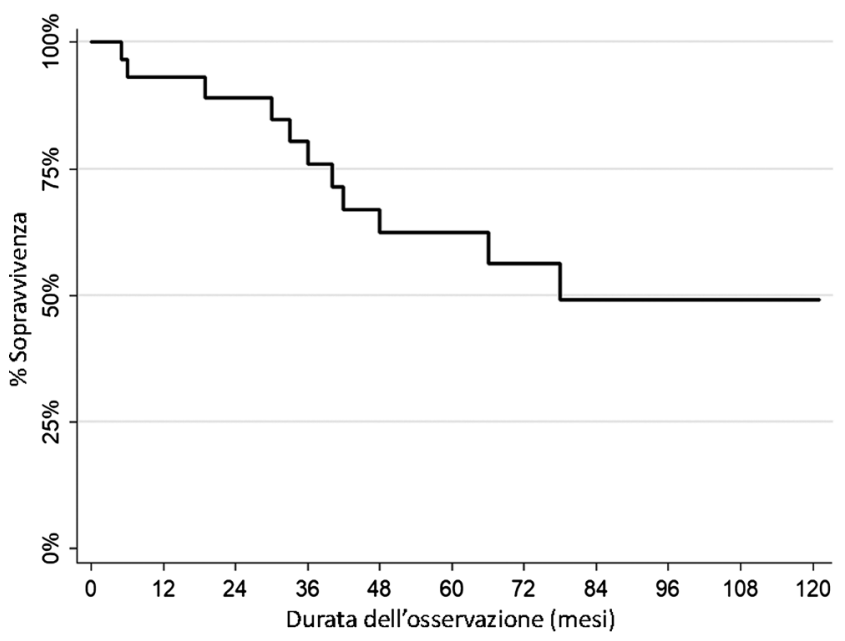

Fig. 1 Sopravvivenza dell'intera casistica di insulinomi maligni

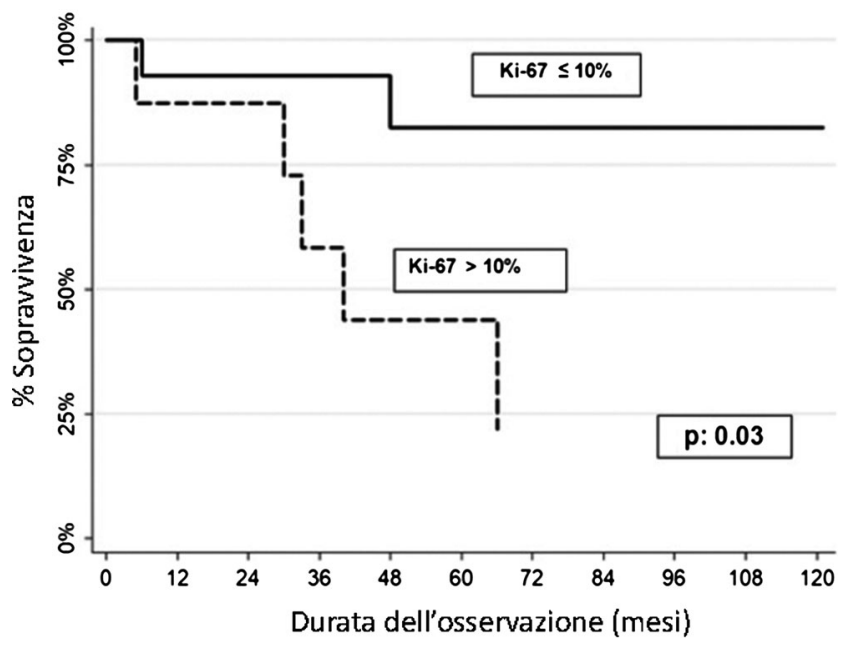

Fig. 2 Sopravvivenza secondo il Ki-67

Questo dato è stato confermato da un'analisi multivariata nella quale il Ki-67 è risultato un fattore prognostico indipendente di sopravvivenza. Nessun paziente con Ki-67 $>10 \%$ era vivo a 10 anni di follow-up (Fig. 2). Un più alto valore di insulina sierica alla diagnosi era associato a una prognosi peggiore. In particolare, è stato riscontrato che i pazienti con insulina all'esordio $\leq 60 \mu \mathrm{IU} / \mathrm{mL}$ avevano una miglior sopravvivenza rispetto a quelli con più alti livelli di insulina (a 5 anni 78 vs 31\%, $p=0,015$ ) (Fig. 3). Per quanto riguarda il tipo di trattamento, i pazienti sottoposti a chirurgia hanno avuto un più alto tasso di sopravvivenza rispetto a chi non ha eseguito l'intervento (a 5 anni del 76 vs 31,7\%, $p=0,006$ ) (Fig. 4).

\section{Conclusioni}

Lo studio multicentrico italiano sugli insulinomi maligni rappresenta una delle più ampie casistiche finora pubbli-

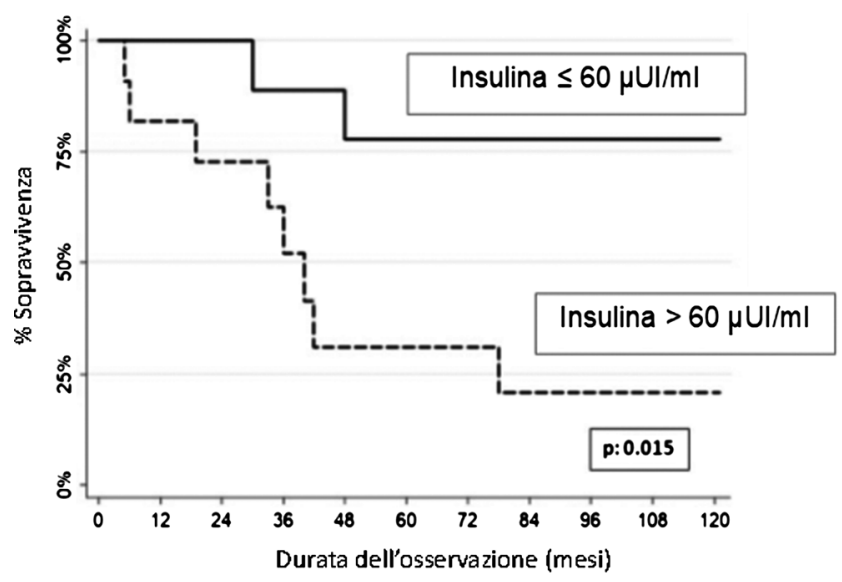

Fig. 3 Sopravvivenza secondo i valori di insulinemia

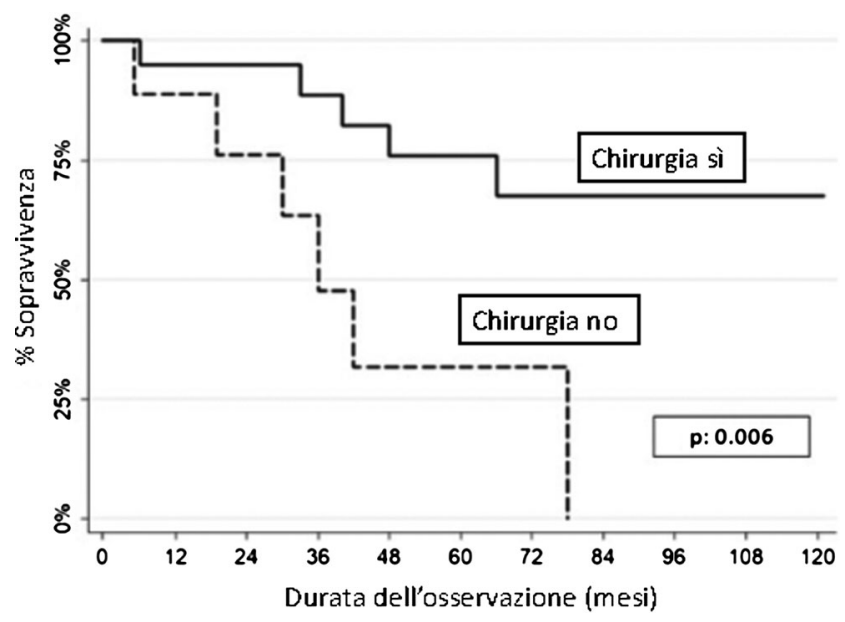

Fig. 4 Sopravvivenza secondo il trattamento chirurgico

cate che riporti dati dettagliati riguardo la presentazione clinica, le caratteristiche tumorali, i trattamenti e i fattori prognostici.

La variabilità di trattamento tra paziente a paziente e tra i vari centri di riferimento per la gestione dei NET riflette la complessità della gestione terapeutica della malattia e la mancanza di linee guida dettagliate sull'argomento.

La sindrome ipoglicemica può rimanere misconosciuta per mesi data l'aspecificità dei sintomi e portare così a un ritardo diagnostico del panNET. Inoltre, può insorgere anche dopo molti anni in un panNET originariamente non funzionante ed essere espressione di una maggior aggressività del tumore.

Gli insulinomi maligni che hanno mostrato una prognosi più favorevole sono quelli sottoposti a intervento chirurgico e con Ki67 $\leq 10 \%$. Anche la severità della sindrome ormonale condiziona la prognosi; infatti, i livelli di insulina $>60 \mu \mathrm{IU} / \mathrm{mL}$ sono associati a un più basso tasso di sopravvivenza. 
La chirurgia, sia con intento curativo che di debulking, è stata la prima scelta di trattamento nella maggioranza dei casi e ha determinato, oltre che un aumento significativo del tasso di sopravvivenza, anche un efficace controllo sintomatico. Tale risultato è in linea con un recente studio retrospettivo basato sul registro americano dei tumori SEER, che ha incluso 121 pazienti con insulinoma maligno, dei quali il $64 \%$ sottoposto a chirurgia [4].

Lo studio multicentrico italiano ha dimostrato, inoltre, come la PRRT sia un trattamento efficace nel controllare la sindrome ipoglicemica nella maggioranza dei pazienti; pertanto, dovrebbe essere effettuata nelle fasi iniziali di progressione di malattia. L'elevata espressione dei recettori della somatostatina di tipo 2 (SSTR-2), confermata dall'alta sensibilità alla ${ }^{68} \mathrm{Ga}$-PET/TC degli insulinomi maligni, li rende candidati alla PRRT.

Infine, il tasso di sopravvivenza a 5 anni è risultato più elevato rispetto a precedenti casistiche $[1,9,10]$ e confrontabile con lo studio basato sul registro americano dei tumori SEER [4]. Il miglioramento della prognosi in queste ultime decadi può essere spiegato dall'avanzamento delle tecniche chirurgiche e dall'introduzione di nuove armi terapeutiche come Everolimus e PRRT.

Data la rarità dell'insulinoma maligno e la complessità della sua gestione terapeutica, è fondamentale l'approccio multi-disciplinare in centri di riferimento.

Funding Note Open access funding provided by Università degli Studi di Verona within the CRUI-CARE Agreement.

Conflitto di interesse Gli autori Alessio Veltroni, Elisa Cosaro e Maria Vittoria Davì dichiarano di non avere conflitti di interesse.

Consenso informato Lo studio presentato in questo articolo non ha richiesto sperimentazione umana.

Studi sugli animali Gli autori di questo articolo non hanno eseguito studi sugli animali.

Nota della casa editrice Springer Nature rimane neutrale in riguardo alle rivendicazioni giurisdizionali nelle mappe pubblicate e nelle affiliazioni istituzionali.

Open Access This article is licensed under a Creative Commons Attribution 4.0 International License, which permits use, sharing, adaptation, distribution and reproduction in any medium or format, as long as you give appropriate credit to the original author(s) and the source, provide a link to the Creative Commons licence, and indicate if changes were made. The images or other third party material in this article are included in the article's Creative Commons licence, unless indicated otherwise in a credit line to the material. If material is not included in the article's Creative Commons licence and your intended use is not permitted by statutory regulation or exceeds the permitted use, you will need to obtain permission directly from the copyright holder. To view a copy of this licence, visit http://creativecommons.org/licenses/by/4.0/.

\section{Bibliografia}

1. Service FJ, McMahon MM, O'Brien PC, Ballard DJ (1991) Functioning insulinoma - incidence, recurrence, and long-term survival of patients: a 60-year study. Mayo Clin Proc 66:711-719

2. Baudin E, Caron P, Lombard-Bohas C et al (2013) Malignant insulinoma: recommendations for characterisation and treatment. Ann Endocrinol (Paris) 74(5-6):523-533

3. Davì MV, Pia A, Guarnotta V et al (2017) The treatment of hyperinsulinemic hypoglycaemia in adults: an update. J Endocrinol Invest 40:9-20

4. Sada A, Glasgow AE, Vella A et al (2020) Malignant insulinoma: a rare form of neuroendocrine tumor. World J Surg 44(7):2288-2294

5. van Schaik E, van Vliet EI, Feelders RA et al (2011) Improved control of severe hypoglycemia in patients with malignant insulinomas by peptide receptor radionuclide therapy. J Clin Endocrinol Metab 96:3381-3389

6. MagalhãesI D, Sampaio L, Ferreira G et al (2019) Peptide receptor radionuclide therapy with 177Lu-DOTA-TATE as a promising treatment of malignant insulinoma: a series of case reports and literature review. J Endocrinol Invest 42(3):249-260

7. Veltroni A, Cosaro E, Spada F et al (2020) Clinico-pathological features, treatments and survival of malignant insulinomas: a multicenter study. Eur J Endocrinol 182:439-446

8. Andreassen M, Ilett E, Wiese D et al (2019) Surgical management, preoperative tumor localization, and histopathology of 80 patients operated on for insulinoma. J Clin Endocrinol Metab 104:6129-6138

9. Lepage C, Ciccolallo L, De Angelis R et al (2010) European disparities in malignant digestive endocrine tumours survival. Int $\mathbf{J}$ Cancer 126(12):2928-3410

10. Camara-de-Souza AB, Toyoshima MT, Giannella ML et al (2018) Insulinoma: a retrospective study analyzing the differences between benign and malignant tumors. Pancreatology 18:298-303 